\title{
Guest-Editorial
}

\section{Special issue: New tendencies on agents and grid environments}

\author{
Pilar Herrero ${ }^{\mathrm{a}}$ and Gonzalo Méndez ${ }^{\mathrm{b}}$ \\ ${ }^{a}$ E-mail: pherrero@fi.upm.es \\ ${ }^{\mathrm{b}}$ E-mail: gmendez@fdi.ucm.es
}

We wish to extend a warm welcome to authors and readers of the Special Issue on "New Tendencies on Agents and Grid Environments".

Web services are a rapidly expanding approach to building distributed software systems across networks such as the Internet. A Web service is an operation typically addressed via a URI, declaratively described using widely accepted standards, and accessed via platform-independent XML-based messages.

Emerging ontologies are being used to construct semantically rich service descriptions. Techniques for planning, composing, editing, reasoning about and analysing these descriptions are being investigated and deployed to resolve semantic interoperability between services within scalable, open environments.

Agents and multi-agent systems can benefit from this combination, and can be used for web service discovery, use and composition. In addition, web services and multi-agent systems bear certain similarities, such as a component-like behaviour, that can help to make their development much easier.

In addition, the increasing development of Service Oriented Architectures (SOA) and Grid Computing are a means to facilitate the combination of agents and web services as a tool to create large-scale distributed systems from heterogeneous sources.

The goal of this special issue is to present a collection of papers which describe, analyze and discuss how to contribute to the development of intelligent web services by merging all these consolidated areas.

All submitted papers presented in this publication have undergone a thoroughly review process with each paper having at least two reviewers providing feedback to the authors. We selected four papers out of more than 26 submissions for this publication.

We would like to thank the members of the Program Committee who gave their time and energy to ensure the high quality of the technical program. We are also grateful to the MAGS editorial board for their support and encouragement. Especially we would like to thank to Prof Rainer Unland for his collaboration and his continuous support.

We acknowledge the efforts of the authors of selected papers for their contributions to the new and exciting interdisciplinary area covered by the publication.

Pilar Herrero

Gonzalo Méndez

Guest Editors 